\title{
Implementation of Web Based Manufacturing Process Planning Workflow in a Pressure Die Casting Industry
}

\author{
M. Nagaraj ${ }^{1}$, Dr.G. Madhan Mohan ${ }^{2}$ \\ $\left({ }^{l}\right.$ PG student, ${ }^{2}$ Assistant professor(Sr.Gr.), Department of Production Engineering, PSG College of \\ Technology, India)
}

\begin{abstract}
Today's manufacturing industry requires product data management system (PDM) for efficient product development and production. Manufacturing industries must deal with competition, time to market, reduction of the cost of doing business, and rapid development of new services and products. To address these requirements industries must constantly reconsider and optimize the way they do business and change their systems and applications to support evolving business processes. In the part of the PDM solution, workflow management system facilitates creating and executing workflow and controlling, monitoring, optimizing and supporting business processes. This paper proposes an approach to implement web based manufacturing process planning workflow in a pressure die casting industry to overcome the bottleneck associated with the workflow of the company. The advantage of this work is its efficiency and flexibility of data management and the business processes are explicitly defined. Manufacturing process planning workflow has been implemented to support the business process of the pressure die casting industry.
\end{abstract}

Keywords: Workflow, Manufacturing Process planning, Workflow management system, Web based application, Change management system

\section{Introduction}

This study aims to discuss the implementation of workflow software in a pressure die casting industry from the point of view of knowledge and information management in product related processes. PDM is seen as a method for companies to manage product development and service processes from beginning to end in product life cycle. Especially in manufacturing companies, managing product information through the whole life cycle is seen as an answer to growing demand in product development. The main problems in the pressure die casting industry are time to market, reducing development cost and maintaining information about all the parts. This research is fully based on a pressure die casting product development process. They are facing a number of problems related to maintaining the product information. The advance technology of the internet, World Wide Web and distributed computing technologies are available for sharing the information over the world. The problem of using the window application is to share the information within the company itself. The web based application in the industry is used for the concept of "Designing in one place, manufacturing in another place". The main aim of the workflow software is to reduce the product development time.

This paper is organized as follows. Section 2 discusses the problem definition. The objective of the research is explained in section 3. Section 4 presents the literature survey. The concept of the workflow is given in section 5. Section 6 provides the overview of the manufacturing process planning workflow. The architecture of the manufacturing process planning workflow is explained in section 7. Section 8 gives the details about user management system. Section 9 describes the plan change management system. Section 10 discusses the conclusion.

\section{Problem Definition}

The pressure die casting industry face various problems like time to market, development cost and new product development.It contains various workflows such as manufacturing process planning workflow, production tooling workflow and manufacturing workflow. They are producing numbers of products for various customers. They have various problems in maintaining the product data. Each product contains all types of data like planning data, design data, resource data, manufactured data and quality data. It eliminates the data integrity, minimizes the consistency, redundancy occurred in the data maintenance. The main problem is the sharing of data between the users involved in the workflow. To overcome all the problems, the workflow software is implemented.

\section{Objective}

The objective of the project is to implement web based manufacturing process planning workflow in a pressure die casting industry. This system can be used to reduce the paper content work. Sharing the data between the organizations will be globalized using web enabling. The business process of the company will be 
improved. The business process of the company explicitly defined so it reduces the time to market of the product. All types of data about product are maintained in the database. Each department workflow has been studied. Based on the study, architecture of the workflow of the department has been drawn. Actors involved in each activity and various tasks have been studied. The use case diagram for all components used in the workflow has been drawn to find out the real problem associated with the system development. Each department workflow related data have been collected to create the web based application. The architecture of the manufacturing process planning workflow has been created and application is developed using a programming language. User management system has been developed for managing the authentication and authorization of using the application. A revision management system was developed for maintaining the changed data for the product. All systems will be integrated using interface concept and then software testing is conducted to analyze the error in the development of the system. Web based manufacturing process planning workflow system is implemented and deployed in the industry to capture error and to find out the reliability of the system.

\section{Literature Overview}

Current literature on workflow management system is mainly focused on large companies with ample resources and their processes. In the literature of SMEs, the workflow management system aspect of business processes has been an increasingly interesting topic, but the research is still scarce. Product data management (PDM) is very important in the domain of product design and development. The product, process and customer process can be seen as the core of all business processes and PDM brings these together by enabling the integration of the all product related information.

Qiu and Wong (2007) described dynamic workflow changes in PDM systems. To facilitate dynamic workflow change, the challenge is to identify all nodes in the new workflow instance to satisfy the following conditions (a) they are unchanged, (b) they have been finished in the old workflow instance and (c) they do not execute again. The proposed approach examined the integration between the workflow and PDM systems. It was suggested that when a workflow instance is about to change, its associated PDM data is to be handled carefully to ensure data integrity.

Kim et al (2006) discussed standards based approaches to B2B workflow integration. Three approaches were used for B2B integration; (a) workflow system interoperability approach, (b) web service choreography approach, and (c) multi-phase process composition approach. The design and implementation of B2B integration were discussed in this paper.

Gimenes et al (2004) presented a component based product line for workflow management systems. The process followed to design the product line was based on the Catalysis method. Extensions were made to represent variability across the process. The domain of workflow management systems has been shown to be appropriate to the application of the product line approach as there are a standard architecture and models established by a regulatory board, the Workflow Management Coalition. In addition, there is a demand for similar workflow management systems, but with some different features. In particular, the design of the workflow execution manager component was described.

Gallet et al (2004) dealt with the use of a UML approach for the specifications of a PDM system (product data management) implementation. A PDM system enables the management of the whole product data and related information about its entire lifecycle. The main goal of this paper is to highlight the added value of using an object-oriented approach for modelling, specifying and implementing a PDM system on a business case study. The chosen object-oriented approach and the used UML diagrams for the modelling and integration of product, process, and resource data was detailed for a turboprop aircraft project.

Aversano et al (2002) presented results and lessons learned from an on-going technology-transfer research project aimed at introducing service and technology innovation within a peripheral public administration while transferring enabling workflow methodologies and technologies to local Small and Medium Enterprises (SMEs). A process reverse engineering approach and its application in the technology transfer project was discussed. They also discussed an approach for evaluation and assessment of workflow technology and present a prototype implementation of a selected process of the subject organization.

Fakasa et al (1999) described the architecture for workflow management systems based on Workflow Intelligent Business Objects (WIBO). The design of the WIBOs is based on principle of intelligence, autonomy, collaboration and co-operation. Using WIBOS that carry out tasks on the user's behalf, it is possible to workflow systems that bring further improvements in process automation and dynamic management, and achieve dynamic reallocation of resources to actors.

Laguna et al (1999) proposed usecases for the favoured technique for defining the functional requirements of a software system, but their use implies that the desired functionality of the new system is well known. The aim of this work was to present an alternative technique and a supporting tool to accurately define this functionality, expressed as use cases, starting from the workflows that describe the end user work. The use of hypergraphs in the proposed algorithm of transformation reinforces the generation process. In addition, the 
technique is independent of the development paradigm and a variation on the algorithm allows obtaining Data Flow Diagrams.

Stoilova et al (1999) provided a short chronology of evolution of workflow management systems. Classifications according to different criteria of software tools supporting modelling and execution functionalities of workflow management systems were presented.

Georgakopoulos et al (1995) provided a high-level overview of the current workflow management methodologies and software products. In addition, the infrastructure technologies that can address the limitations of current commercial workflow technology and extend the scope and mission of workflow management systems to support increased workflow automation in complex real-world environments involving heterogeneous, autonomous, and distributed information systems were discussed. In particular, how distributed object management and customized transaction management can support further advances in the commercial state of the art in this area was discussed.

\section{Workflow}

Workflow is a new information processing technology that helps implementing business process that can easily adapt to the changing needs of a dynamic environment. It is sometimes defined as the computerized automation of the business process. The work processes performed within a business organization are the basic units of that organization. In the business world, a process refers directly to a business activity and its corresponding information process. Workflow management involves everything from defining and modeling processes to synchronize the activities of people or applications that perform that process. A process model describes the structure of a business process (such as order entry or approval process) in the real world. It must define all the possible paths that may be taken and include rules that define when the various paths and actions are to be performed or taken. The diagram for the workflow is shown in Fig.1.

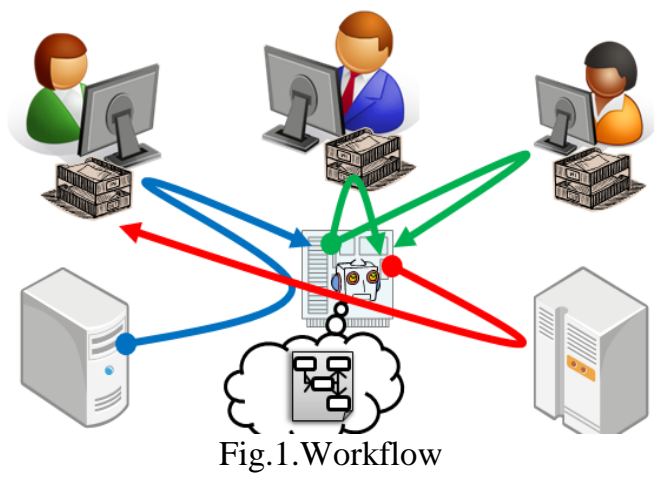

\section{Manufacturing Process Planning Workflow}

The manufacturing process planning workflow is very important in the product development process in a pressure die casting industry. It is used to analyze the feasibility of part production. The customer can send the all details about the part to the company. These details will be verified by the customer representative and then sent to the planning department. The manufacturing process planning workflow is shown in Fig.2. The details of the part will be rejected by him, if the information sent by the customer is not true. The information about the

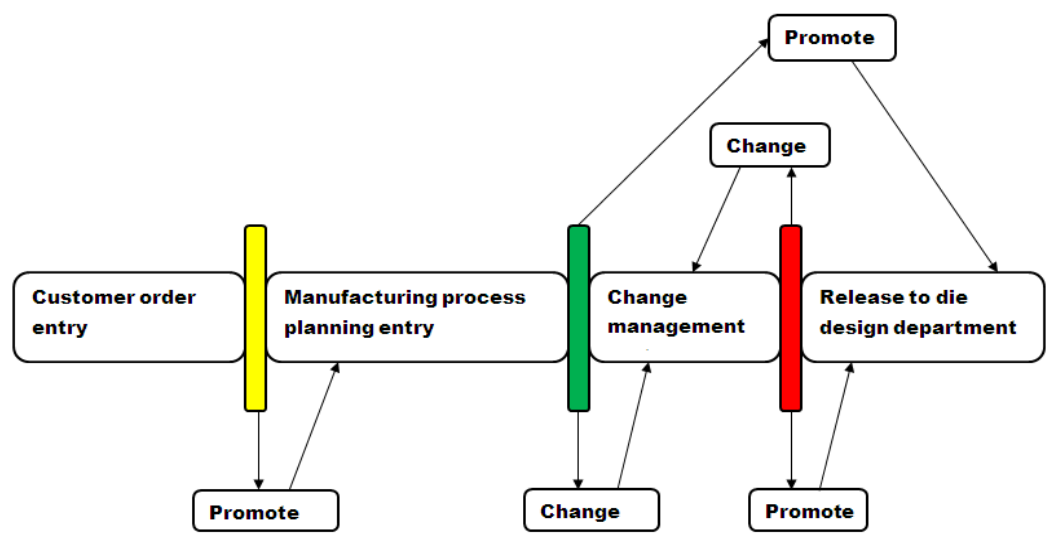

Fig.2. Manufacturing process planning workflow 
part will be received from the process manager and then he will send the details to the planning department. The planning on the part will be completed by all users. Manufacturing process planning data entry is used to submit and store all the planning data of the product in the database. The usecase diagram of the manufacturing process planning entry system is shown in Fig.3.Various planning forms are available in the MPP entry like Manufacturing feasibility report, Resource requirements and fulfillment form, Corrective action for machine capacity utilization report, Risk analysis report and Contract review check entry report.

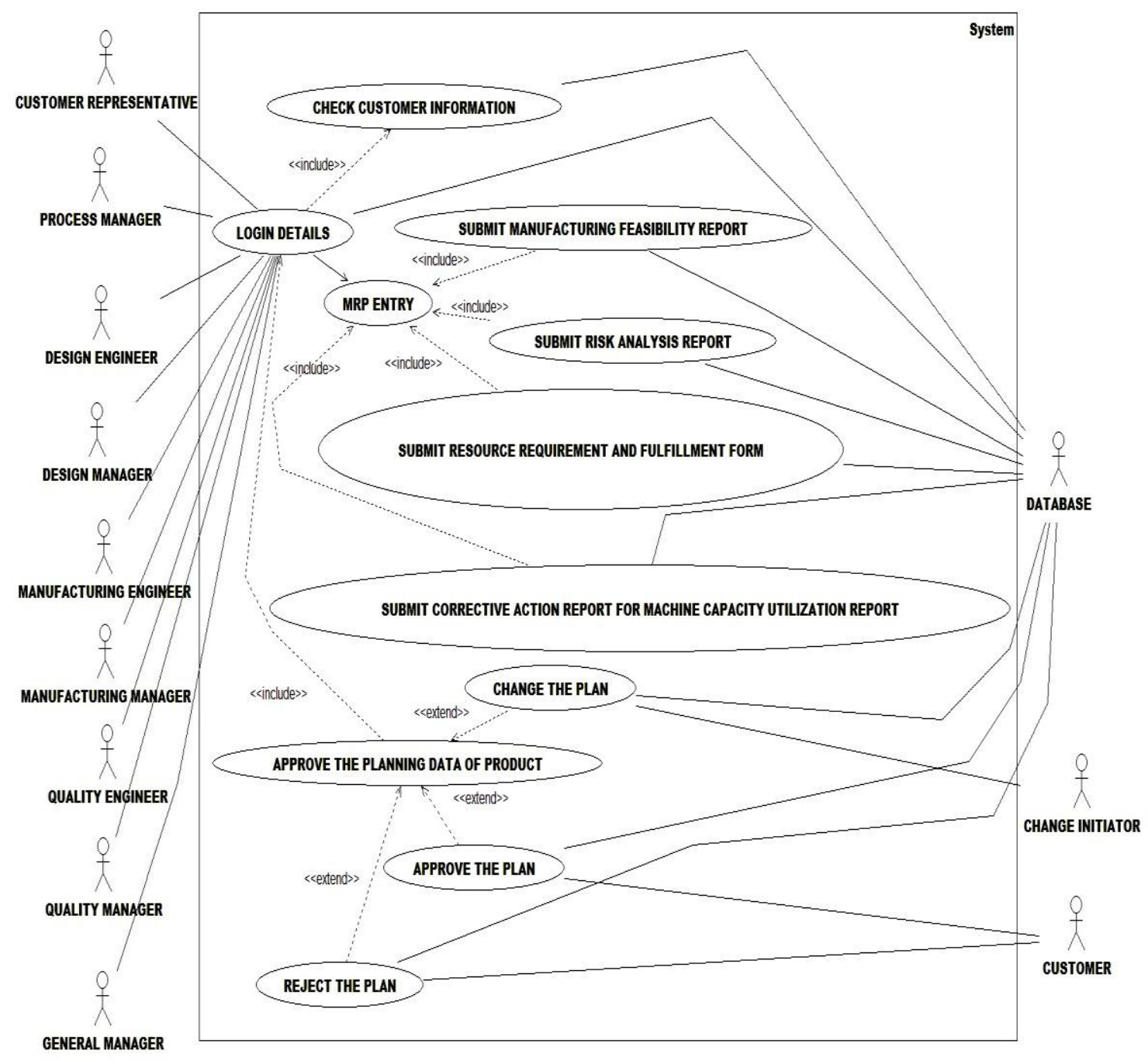

Fig.3.Use case diagram of the manufacturing process planning data entry system

The activity diagram of the manufacturing process planning workflow is shown in Fig.4. It consists of three major tasks such as customer order entry, manufacturing process planning entry and change management system. Here the customer is called as tier 1 companies. Initially, the customer order entry task is performed by the customer that will be reviewed by the customer representative in the industry. Every task output is taken as input to another task and finally produces workflow output. When the details is not feasible for the production of part they will be rejected by the industry. If the details are feasible for it they will be sent to process planning department. Process planning on the part is done by the planning department and they will enter all details of planning related data. It consists of five subtasks such as manufacturing feasibility report, risk analysis report, requirement fulfillment report, machine capacity utilization report and contract review report. All planning details are reviewed by the process manager and approved by the general manager of the company. If any changes are required in the planning report they will be performed by the CFD or otherwise the initial planning report is accepted and sent to the die design department. Change management has three sub tasks such as change request, change request review and change request approval. The changes in the change of plan are also approved by the general manager. Finally, all details related to the planning of the product are sent to the die design department. 


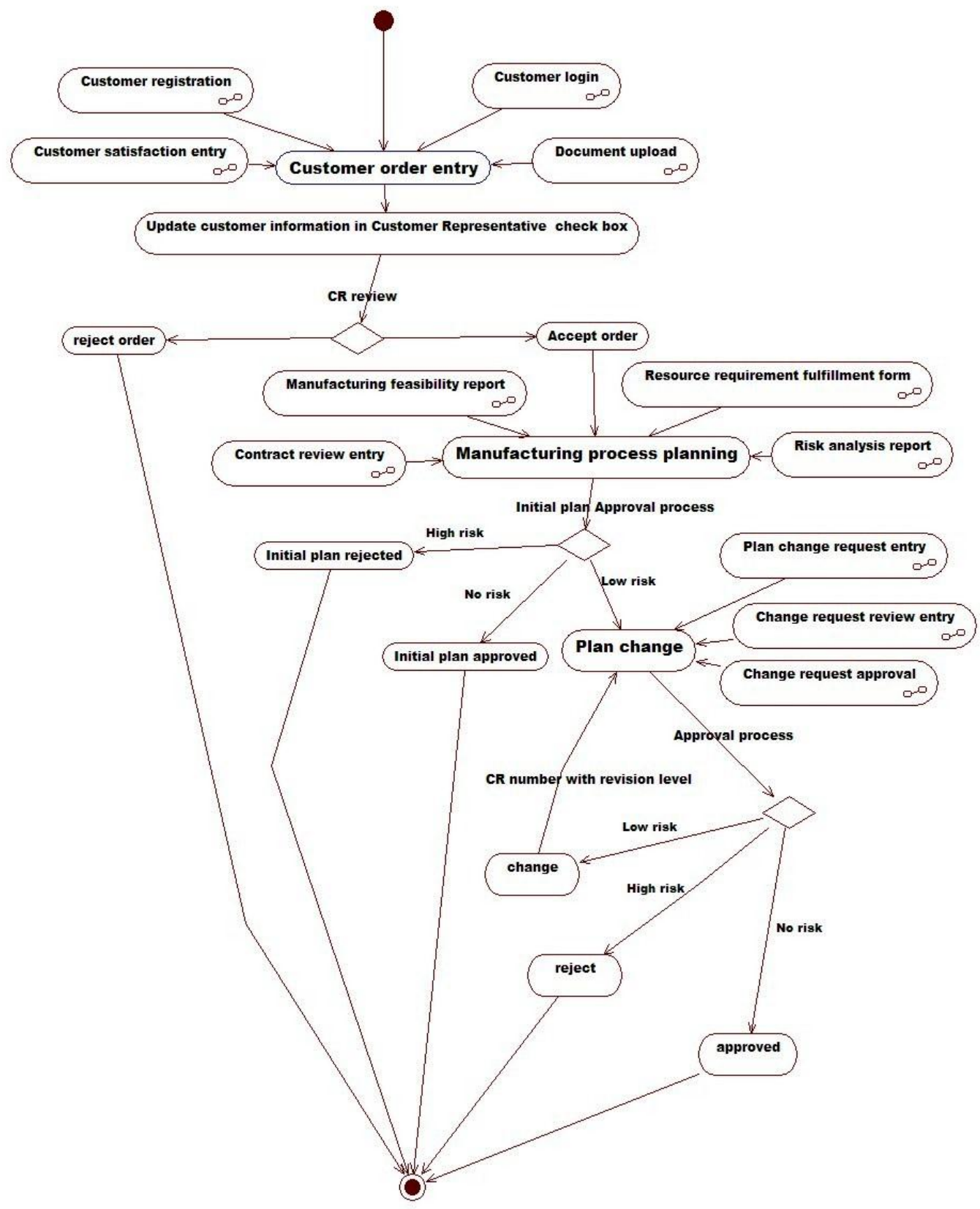

Fig.4. Activity diagram of the manufacturing process planning workflow

The planned data will be sent to the general manager who has responsibility for approving the plan for the product and finally he decides whether the part is feasible to the production or not. Then the contract review list will be sent to the design department for the die design. Any changes that are required on the part will be completed by using change management system. The data of the changed plan are maintained in the separate database which is called as revision data management system. The change of a product will be done by the Cross Functional Team (CFT). The changed contract review proposal will be passed to the die design department.

\section{Architecture For Development Of Manufacturing Process Planning Workflow}

The planning workflow management system is implemented as a web application by using three tier architecture concepts. The architecture for development of web based process planning workflow is shown in Fig.5. The web server contains all the objects of workflow systems. Based on the request from the user, the web server gives the responses to the client. The planning templates are objects which are available on the web server. A data lifecycle management module enforces data history management and a user management system 
module enforces data access control. The change management system is used to manage the execution of changes in the plan from which the changed data will be generated and then it will be stored in the revision plan data management system. The web page of the manufacturing process planning entry is shown in Fig.6.

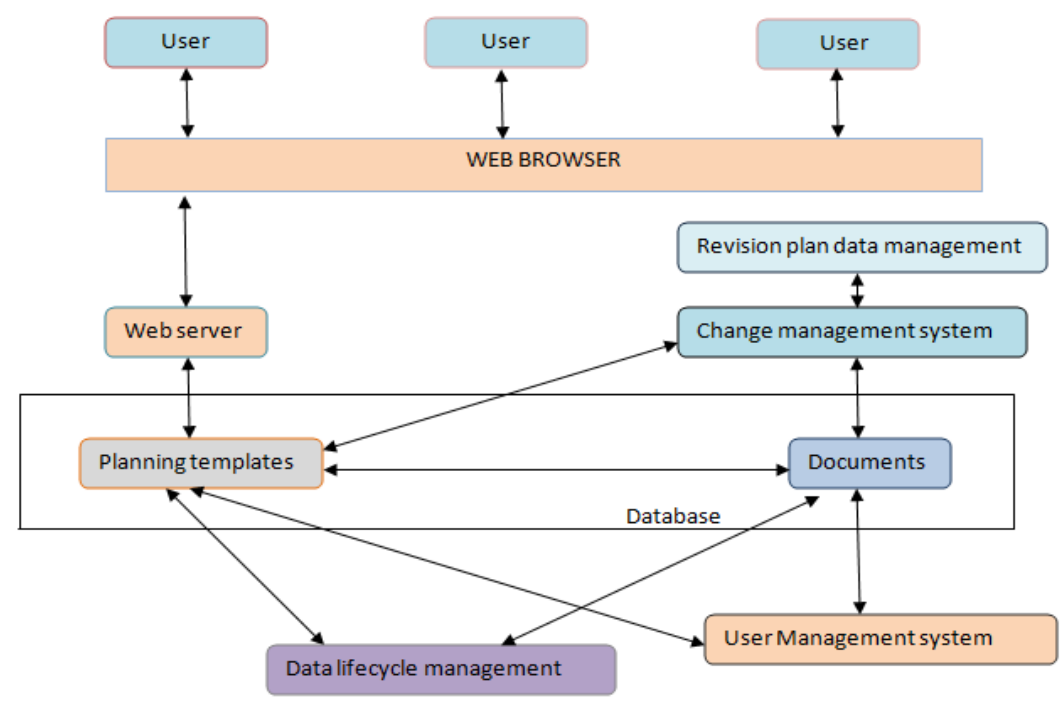

Fig.5. Architecture of the MPPW

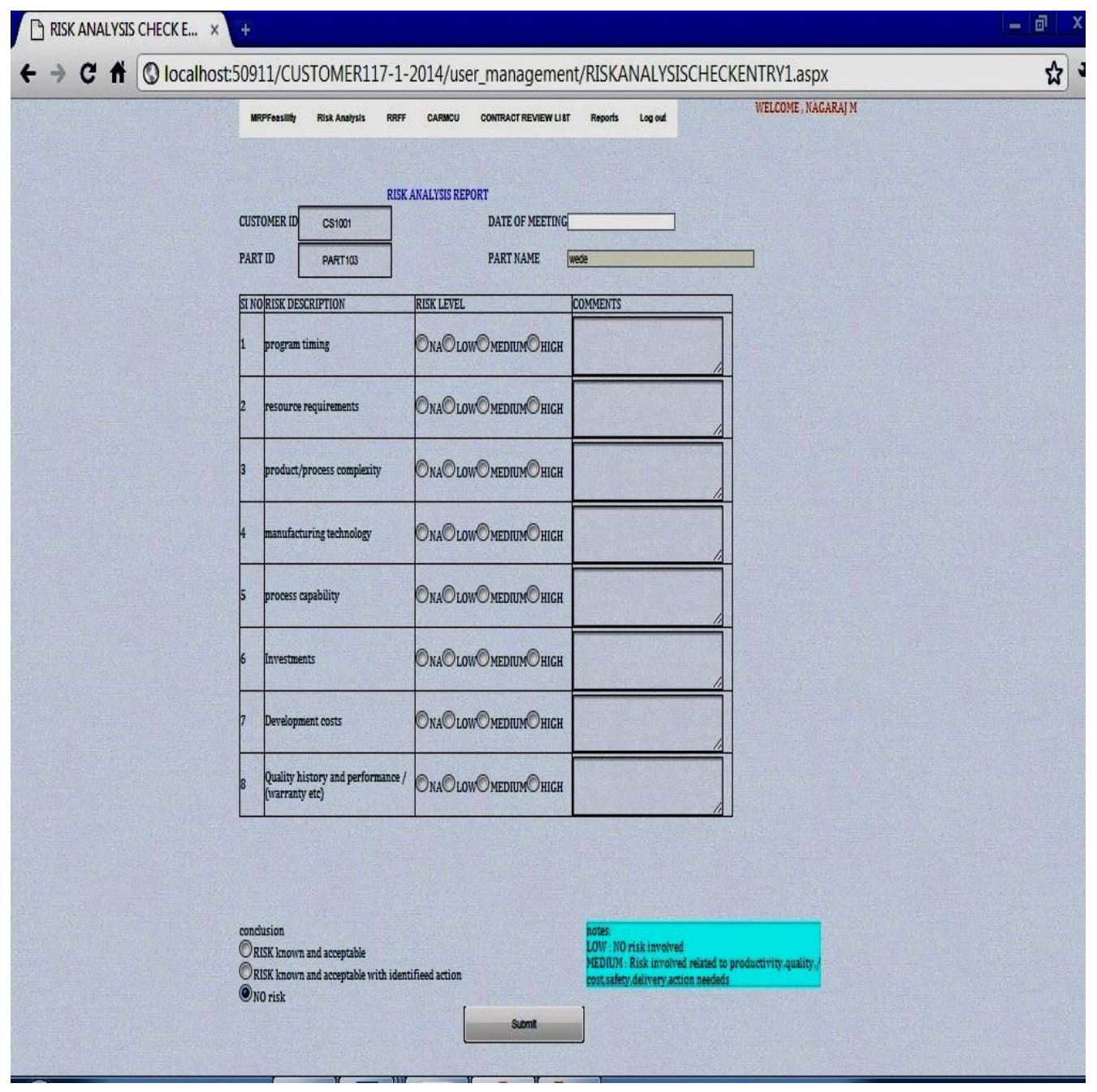

Fig.6.Web page of the manufacturing process planning entry system 


\section{User Management System}

The User Management System provides functionality to manage users and personal profiles. It is reliable, extensible and open for easier integration with existing systems. UMS implements user management and authentication, personal profiles management and classification. Personal profiles contain information, such as profile identification number, email, first and last name, etc. Personal profiles are used for personal information such as names, addresses, etc. In order to access personal profile for reading and modification PIN number is used for authentication. The usecase diagram of the user management system is shown in Fig.7.User information contains user identification number, username, password and session for authentication. User information is used for high-level authentication and privilege definitions. One or more personal profiles are assigned to a user. In addition, there are user groups for the purpose of group specific privileges and profile roles for application specific need related to the user's role in the system. Based on the position of the person, an identification number will be generated. The forms which contain the task for the role of each activity will be selected by unique identification number and position of the employee.

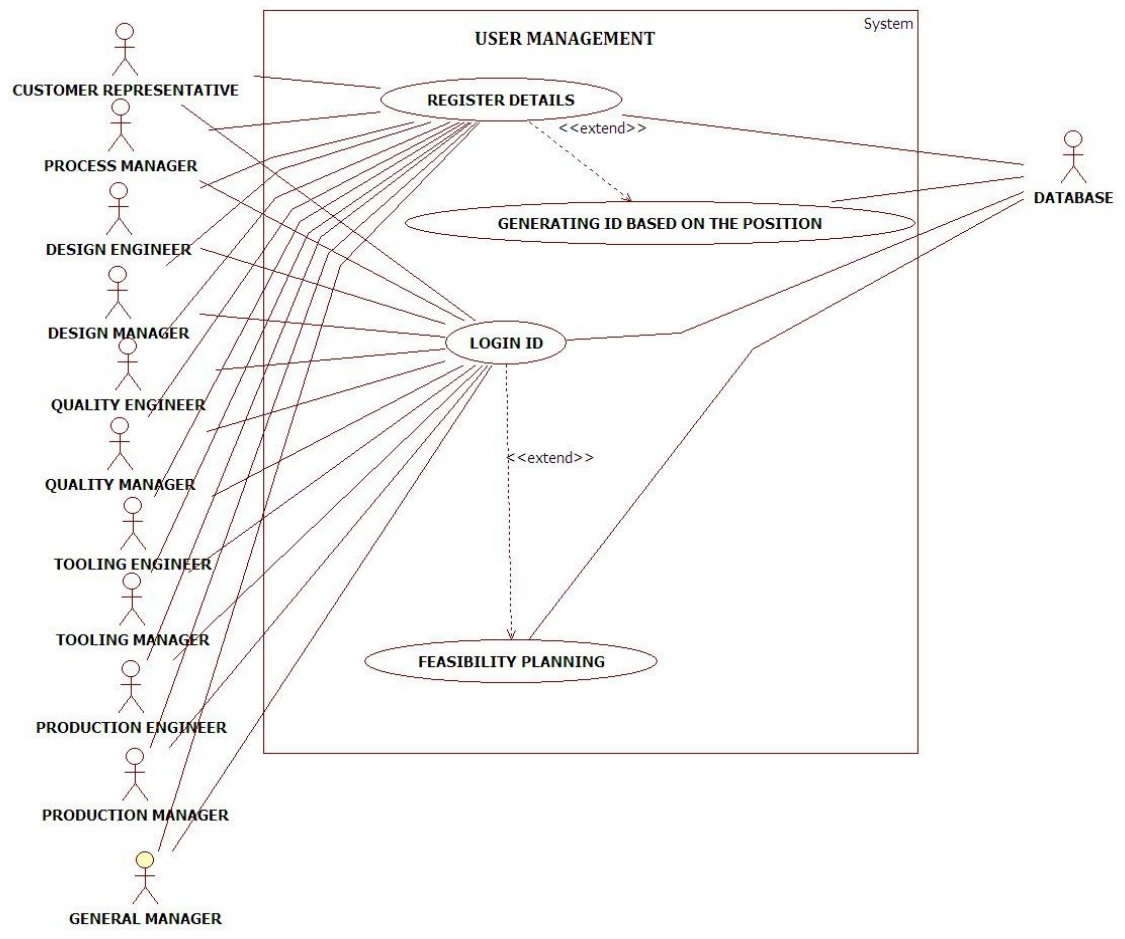

Fig.7. Usercase diagram of the user management system

\section{Change Management System}

A Change Management Process is a method by which changes to the plan (e.g. With the scope, deliverables, timescales or resources) are formally defined, evaluated and approved prior to implementation. The process entails completing a variety of control procedures to ensure that, if implemented, the change will cause minimal impact to the objectives of the planning. Any change to the planning during the Execution phase will need to be formally managed as part of the Change Management Process. Without a formal Change Management Process in place, the objective of delivering a solution within 'time, cost and quality' may be compromised. The plan Change Management Process is terminated only when the Execution phase of the planning is completed. The usecase diagram of the plan change management system is shown in Fig.8. Three main steps in the change management system like (i) Change request, (ii) Change request review and (iii) Change request approval.

\subsection{Change Request Form}

The Change Request Form is used to identify and describe a proposed change in the planning. This process provides the ability for any member of the planning team to submit a request for a change to the planning. The Change Initiator identifies a requirement for change to any aspect of the planning (e.g. Scope, deliverables, timescales and organization). He completes a Change Request Form (CRF) and distributes the form to the Cross Functional Team. He initially recognizes a need for change to the planning and formally communicates this requirement to the General Manager. He is responsible for identifying the requirement to make a change to the planning, documenting the requirement by completing a CRF and submitting the CRF to the General Manager for review. 


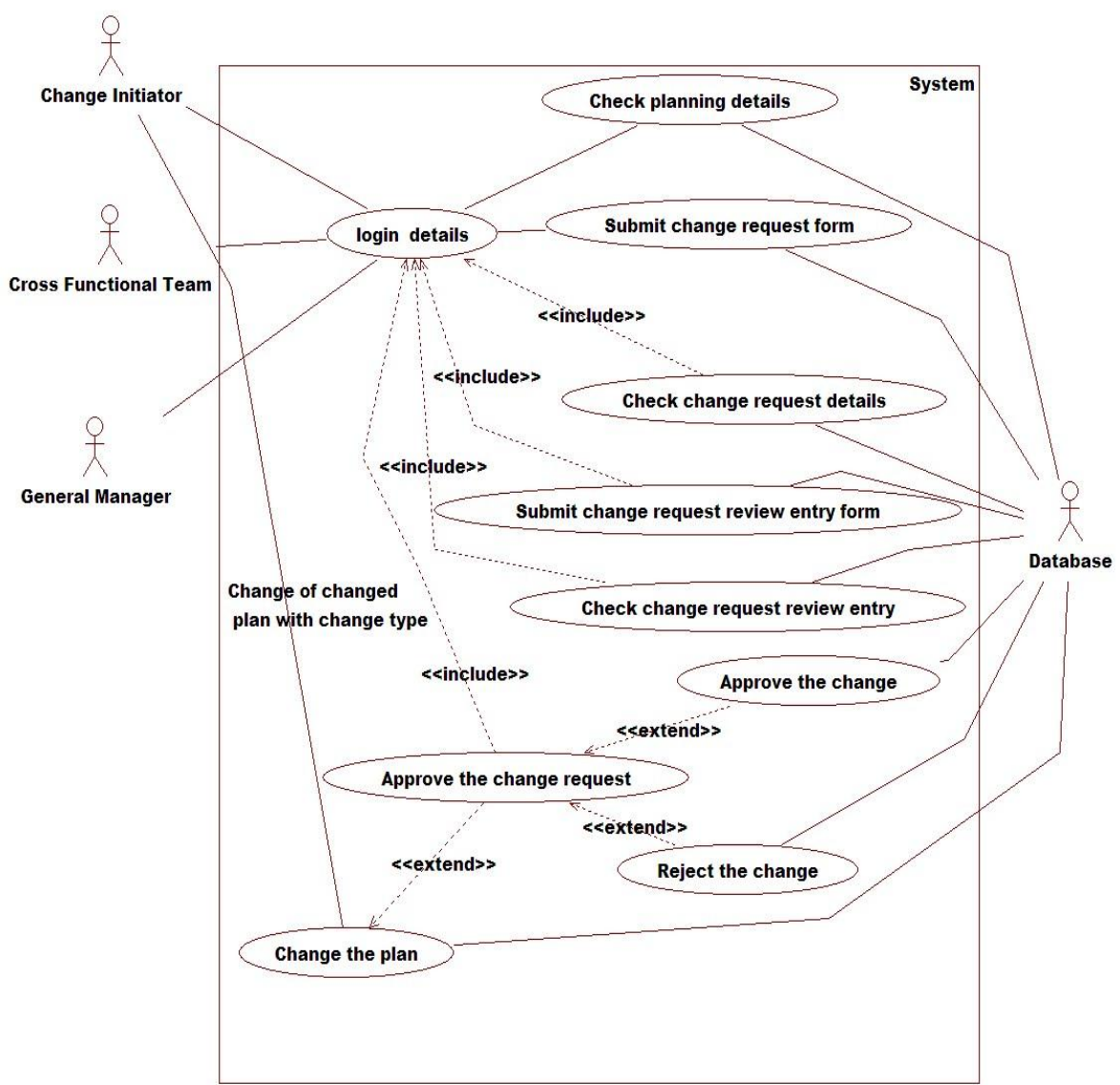

Fig.8. Usecase diagram of the change management system

\subsection{Review Change Request}

The General Manager reviews the CRF and determines whether or not additional information is required for the Cross Functional Team to assess the full impact of the change of the planning time, scope and cost. The decision will be based on factors, such as the number of change options presented, Feasibility and benefits of the change, Complexity and/or difficulty of the change options requested and the scale of the change solutions proposed. The General Manager will record the CRF details in the Change Log to track the status of the change request. The Cross Functional Team (CFT) is the Steering Committee or other authorized body who is the principal authority for all CRFs forwarded by the General Manager. The CFT is responsible for reviewing all CRFs forwarded by the General Manager, considering all supporting documentation, approving / rejecting each CRF based on its relevant merits, resolving change, conflict and identifying the implementation timetable (for approved changes).

\subsection{Approve Change Request}

The General Manager will forward the Change Request Form and any supporting documentation to the Cross Functional Team (CFT) for review and final approval. The 'Change Log' is the log where all requests for changes are registered and tracked through to resolution. The CFT will determine the feasibility of this change by examining factors, such as risk to the planning in implementing the change, risk to the planning in not implementing the change and impact on the planning in implementing the change (time, resources, finance, quality).After a formal review, the CFT may reject the change, request more information related to the change, approve the change as requested and approve the change subject to specified conditions. If the change is approved, the following will occur such as an implementation date of the change will be identified, a test of the change will be scheduled and performed, the change will be implemented, the implementation of the change will be reviewed and deemed successful or corrective actions taken, the success of the change implementation will be communicated to all parties and the change request will be closed on the Change Log. 


\section{Conclusion}

New product development is a very complex and time consuming process in an industry. During this phase, large amount of data are generated. The pressure die casting industry has various problems in managing the data. A workflow has been created using software which eliminates the complication arised during the data management. A web based application enables the data sharing around the globe. Change management system manages the changes associated with the process planning. The user management system provides not only the authentication and integrity of the task, but also the logical relationship between the industries. Manufactuirng process planning workflow is deployed in a industry to improve the business process. The advantage of this work is its efficiency and flexibility of data management and the business processes are explicitly defined.

\section{Acknowledgement}

The authors express their sincere thanks to the M/s Excel Diecastings, Chennai for providing the necessary support.

\section{References}

[1]. Qui.Z.M, and Wong.Y.S., Dynamic workflow change in PDM systems", Computer in Industry, Vol.58 (2007) 453-563.

[2]. Jae-Yoon Jung, Hoonate Kim, and Suk-Ho Kang., Standards based approaches to B2B integration, Computer \& Industrial vol. (2006) 321-334.

[3]. Itana Maria de Souza Gimenes, Fabrício Ricardo Lazilha, Edson Alves de Oliveira Junior, Leonor Barroca, A Component basedProduct Line for Workflow Management Systems, CLEI electronic journal, vol. 7(2004) paper 5.

[4]. Eynard, Thomas Gallet, Pierre Nowa, and Lionel Roucoules., UML based specifications of PDM product structure and workflow, Computers in Industry 55 (2004) 301-316.

[5]. [Miguel A. Laguna, Jose M. Marque, and Francisco J. Garcia, DocFlow: workflow based requirements elicitation, Information and Software Technology 45 (2003) 357-369.

[6]. Lerina Aversano, Gerardo Canfora , Andrea De Lucia, and Pierpaolo Gallucci., Business process reengineering and workflowautomation:a technology transfer experience, The Journal of Systems and Software 63 (2002) 29-44.

[7]. Krasimira P. Stoilova , and Todor A. Stoilov., Evolution of the workflow management systems, Information system, vol.28 (1999) 27-60.

[8]. G.Fakasa, and B.Karakotas ., A workflow management system based on intelligent collaborative products, Information andSoftware Technology, vol.51(1999) 907-915.

[9]. Diimitrios Georgakopoulos, Mark Hornick., An Overview of Workflow Management: From Process Modeling to Workflow Automation Infrastructure, Distributed and Parallel Databases, 3(1995) 119-153. 